\title{
Die Stellung der Radiologie in der ambulanten spezialfachärztlichen Versorgung nach $§ 116 b$ SGB V
}

\section{Einführung \\ $\nabla$}

Mit dem GKV-Versorgungsstrukturgesetz (GKV-VStG), welches zum 01.01.2012 in Kraft getreten ist, hat der Gesetzgeber die bisherige „ambulante Behandlung im Krankenhaus" nach § 116b SGB V durch die „ambulante spezialfachärztliche Versorgung “(ASV) abgelöst, die als neue sektorenübergreifende Versorgungsform nun eine Zusammenarbeit von niedergelassenen Vertragsärzten und Krankenhäusern unter gleichen Voraussetzungen und Teilnahmebedingungen vorsieht.

Allerdings hat der Gesetzgeber den sachlichen Anwendungsbereich der Vorschrift deutlich eingegrenzt. Während bis Ende 2011 der Katalog zur ambulanten Behandlung „hochspezialisierte Leistungen, seltene Erkrankungen und Erkrankungen mit besonderen Krankheitsverläufen“ umfasste, sieht die Neuregelung nunmehr in $\S 116 \mathrm{~b}$ Abs. 1 Satz 2 SGB V nur noch

„schwere Verlaufsformen von Erkrankungen mit besonderen Krankheitsverläufen, seltene Erkrankungen und Erkrankungszustände mit entsprechend geringen Fallzahlen sowie hochspezialisierte Leistungen“

als Gegenstand der ASV vor. Von § 116b SGB V umfasst sind nach Abs. 1 Satz 1 SGB $\mathrm{V}$ „die Diagnostik und Behandlung komplexer, schwer therapierbarer Krankheiten, die je nach Krankheit eine spezielle Qualifikation, eine interdisziplinäre $\mathrm{Zu}$ sammenarbeit und besondere Ausstattungen erfordern“. Diese Begrenzung der Anwendungsbereiche der ASV hat zur Folge, dass damit deutlich mehr Leistungsfälle innerhalb der vertragsärztlichen Regelversorgung verbleiben und Patienten, die z.B. an einer onkologischen Erkrankung leiden, erst ab einem bestimmten Erkrankungsbild der Zugang zur ASV gewährt wird.

Zur Konkretisierung der genannten Erkrankungen und zur Bestimmung des Behandlungsumfanges ist nach $\S 116 \mathrm{~b}$ Abs. 4 SGB V der Gemeinsame Bundesaus- schuss (G-BA) beauftragt worden. Das bedeutet, dass der G-BA im Rahmen seiner Beschlüsse auch festlegen muss, ab welchem Krankheitsstadium der Patient in der ASV behandelt werden darf. Er hat im Rahmen der Richtlinie über die ambulante spezialfachärztliche Versorgung (ASVRL) auch die Teilnahmevoraussetzungen und die sächlichen und personellen Anforderungen für Vertragsärzte und Krankenhäuser festgelegt.

Mittlerweile hat der G-BA mit dem Beschluss der „Anlage 2 a) Tuberkulose und atypische Mykobakteriose" vom 19.12.2013 und dem Beschluss der „Anlage 1 a) onkologische Erkrankungen - Tumorgruppe 1: gastrointestinale Tumoren und Tumoren der Bauchhöhle“ vom 20.02.2014 erste Konkretisierungen vorgenommen. Als nächste Erkrankungen sind im Bereich der Onkologie die gynäkologischen Tumoren, einschließlich des Mammakarzinoms und rheumatologische Erkrankungen in Vorbereitung.

Die ASV erfordert eine Zusammenarbeit der beteiligten Ärzte im Rahmen eines interdisziplinären Teams und den Abschluss von Kooperationsverträgen zwischen den Ärzten untereinander und mit den Krankenhäusern. Zur Umsetzung der ASV wurden im Rahmen der ASV-RL durch den G-BA eigenständige Organisations- und Kooperationsvorgaben geschaffen, die nur für diesen Leistungsbereich gelten und von den beteiligten Leistungserbringern zu beachten sind, um die Teilnahmevoraussetzungen der ASV zu erfüllen.

Als problematisch wird seitens der Deutschen Röntgengesellschaft empfunden, dass das Fachgebiet der Radiologie, durch den G-BA bei der Ausgestaltung der personellen Anforderungen ( $\$ 3$ ASV-RL) und des Behandlungsumfanges ( $§ 5$ ASV-RL) im Rahmen der bisherigen Konkretisierungen (gastrointestinale Tumoren, Tuberkulose) nur als hinzuzuziehendes Fachgebiet aufgenommen worden ist. Diese Form der Einbeziehung der Radiologie innerhalb des interdisziplinären Teams hat zur Folge, dass eine unmittelba-

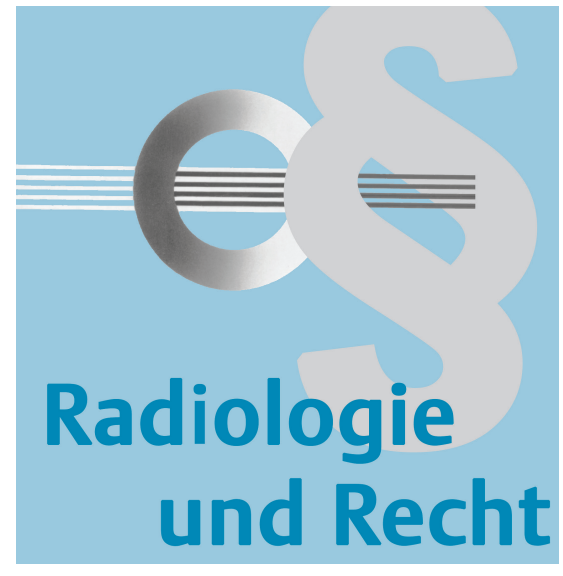

re Beteiligung der radiologischen Diagnostik bei diesen Erkrankungen nicht mehr gegeben ist und die Inanspruchnahme nur im Rahmen eines Definitionsoder Indikationsauftrages möglich ist, der dem Radiologen kaum Therapieoptionen belässt. Bei den aktuell zur Beratung anstehenden gynäkologischen Tumoren besteht die Gefahr, dass der Radiologe auch für das Mammakarzinom nur als hinzuzuziehendes Fachgebiet definiert wird, obwohl die Diagnostik dieser onkologischen Erkrankung zum Kernbereich der Radiologie gehört.

Um die derzeitigen Kooperationsmöglichkeiten für Radiologen in der ASV zu erläutern, werden nachfolgend die rechtlichen Anforderungen an die Zusammenarbeit im interdisziplinären Team und die notwendigen Inhalte der Kooperationsverträge dargestellt und erläutert (Punkt I.). Darüber hinaus wird in einem weiteren Schritt dargelegt, warum die Entscheidung des G-BA, das Fachgebiet der Radiologie nur als hinzuzuziehendes Fachgebiet in das interdisziplinäre Team einzubinden, sachwidrig erscheint und für die Patienten negative Folgen im Behandlungsablauf haben kann (Punkt II.)

\section{Begriffsbestimmungen}

$\nabla$

Für die Teilnahme an der ASV sind die gesetzliche Regelung in $\S 116 \mathrm{~b}$ SGB V und Richtlinien durch den G-BA geschaffen worden. Um an der ASV teilzunehmen, müssen bestimmte Teilnahmevoraussetzungen erfüllt sein. $\mathrm{Zu}$ beachten sind aktuell:

1. Richtlinie die ambulante spezialfachärztliche Versorgung § 116 b SGB V (ASV-RL) vom 21.03.2013. 
2. Anlage $2 \mathrm{a}$ ) Tuberkulose und atypische Mykobakteriose vom 19.12.2013.

3. Anlage 1a) onkologische Erkrankungen - Tumorgruppe 1: gastrointestinale Tumoren und Tumoren der Bauchhöhle vom 20.02.2014 (noch nicht in Kraft).

4. Vereinbarung gemäß § $116 \mathrm{~b}$ Abs. 6 Satz 12 SGB V über Form und Inhalt des Abrechnungsverfahrens sowie die erforderlichen Vordrucke für die ambulante spezialfachärztliche Versorgung (ASV-AV) vom 17.03.2014.

Zum besseren Verständnis über die Arten der Zusammenarbeit in der ASV werden hier die wesentlichen Begriffsbestimmungen erläutert.

ASV-Berechtigte ( $§ 2$ Abs. 1 ASV-RL) Die Leistungen der ASV können von Leistungserbringern, die an der vertragsärztlichen Versorgung nach $\S 95$ Abs. 1 S. 1 SGB V teilnehmen, also von Vertrags(zahn-) ärzten, Psychotherapeuten und MVZ sowie von zugelassenen Krankenhäusern nach § 108 SGB V erbracht werden, soweit sie die Anforderungen und Voraussetzungen nach der Richtlinie erfüllen (ASV-Berechtigte).

Soweit teilnehmende Leistungserbringer eine freiwillige oder verpflichtende Kooperation zur Erfüllung der Anforderungen und Voraussetzungen der ASV-RL eingehen, bleibt ASV-Berechtigter im Sinne der ASV-RL der einzelne Leistungserbringer, der seine ASV-Leistungen im Rahmen der Kooperation eigenständig erbringt ( $\S$ 2 Abs. 1 ASV-RL). Auch wenn damit die ASV-Berechtigten die von ihnen erbrachten Leistungen jeweils eigenständig gegenüber der Krankenkasse abrechnen und nicht das interdisziplinäre Team ( 22 Abs. $2 \mathrm{ASV}-\mathrm{AV}$ ), ist angesichts der gemeinsamen Zweckverfolgung (gemeinsame interdisziplinäre Behandlung im Team) davon auszugehen, dass durch den Zusammenschluss der Vertragspartner eine Gesellschaft bürgerlichen Rechts nach $\S 705$ $B G B$ begründet wird.

Dies hat zur Folge, dass die Vertragspartner gesamtschuldnerisch für etwaige Behandlungsfehler gegenüber dem Patienten haften. Die gesamtschuldnerische Haftung gilt auch für die vertragliche Haftung gegenüber anderen Vertragspartnern und bei Regressen der Krankenkassen. Es sind daher in dem Kooperations- vertrag Regelungen zu treffen, die eine wechselseitige Haftungsfreistellung der Vertragspartner zumindest im Innenverhältnis sicherstellen. Im Außenverhältnis kann demgegenüber die gesamtschuldnerische Haftung ohne Zustimmung der Gläubiger nicht wirksam abbedungen werden.

Interdisziplinäres Team

(§ 3 Abs. 1 ASV-RL)

Die ASV erfordert regelmäßig die Zusammenarbeit in einem interdisziplinären Team, soweit in den Anlagen nichts Abweichendes geregelt ist ( $§ 2$ Abs. 1 ASV$\mathrm{RL}$ ). Abweichende Regelungen sind insoweit möglich, bei denen allein die besondere Expertise eines einzelnen Fachgebietes oder besondere technische Anforderungen die Zughörigkeit zur ASV begründen und dies entsprechend in den Konkretisierungen definiert ist.

\section{Zusammensetzung des interdiszip-} linären Teams (§ 3 Abs. 2 ASV-RL) Das interdisziplinäre Team besteht aus einem Teamleiter (Teamleitung), dem Kernteam und bei medizinischer Notwendigkeit zeitnah hinzuzuziehender Fachärzte.

\section{Teamleitung}

Die Teamleitung hat die Aufgabe, die spezialfachärztliche Versorgung der Patienten fachlich und organisatorisch zu koordinieren. Sie gehört dem Kernteam an. Insoweit nimmt die Teamleitung eine Sonderstellung im Rahmen des interdisziplinären Teams ein.

\section{Kernteam}

Die Mitglieder des Kernteams sind Fachärzte, deren Kenntnisse und Erfahrungen zur Behandlung in der Regel eingebunden werden müssen. Sie müssen die spezialfachärztlichen Leistungen grds. am Tätigkeitsort der Teamleitung, mindestens aber an einem Tag in der Woche zu festgelegten Zeiten am Tätigkeitsort der Teamleitung erbringen. An immobile Apparate gebundene Leistungen sowie die Aufbereitung und Untersuchung von bei Patienten entnommenem Untersuchungsmaterial sind hiervon ausgenommen. Der Ort der Leistungserbringung für direkt an dem Patienten zu erbringende Leistungen muss dennoch in angemessener Entfernung (in der Regel in 30 Minuten) vom Tä- tigkeitsort der Teamleitung erreichbar sein.

Die Zusammensetzung des Kernteams wird durch den G-BA für jede Konkretisierung in den Anlagen neu festgelegt. Im Anzeigeverfahren sind die Mitglieder der Teamleitung und des Kernteams namentlich zu benennen ( $\$ 2$ Abs. 2 ASV-RL).

\section{Hinzuzuziehende Fachärzte}

Die hinzuzuziehenden Fachärzte sind solche, deren Kenntnisse und Erfahrungen in Abhängigkeit vom jeweiligen Krankheitsverlauf typischerweise bei einem Teil der Patienten ergänzend benötigt werden. Ihr Tätigkeitsort für direkt an dem Patienten zu erbringende Leistungen muss in angemessener Entfernung (in der Regel in 30 Minuten) vom Tätigkeitsort der Teamleitung erreichbar sein.

Im Anzeigeverfahren sind die hinzuzuziehenden Fachärzte grds. als Institution (z.B. MVZ) oder namentlich (z.B. Einzelpraxis) zu benennen ( $§ 2$ Abs. 2 ASV-RL).

\section{Leistungskooperationen}

(§ 3 Abs. 1 ASV-RL)

Die Erfüllung der personellen oder sächlichen Anforderungen im interdisziplinären Team nach der ASV-RL und den Anlagen kann auch im Rahmen von vertraglich vereinbarten Kooperationen erfolgen. Im Rahmen des Anzeigeverfahrens nach $\S$ 116b Absatz 3 Satz 1 SGB V ist der Nachweis vertraglicher Vereinbarungen über Leistungskooperationen gegenüber dem erweiterten Landesausschuss zu erbringen ( $§ 2$ Abs. 2 ASV-RL). Der Abschluss eines Kooperationsvertrages über eine Leistungskooperation kann innerhalb eines Sektors (intrasektoral) oder sektorenübergreifend (intersektoral) erfolgen $(\S 3$ Abs. 1 S. 2 ASV-RL).

\section{ASV-Kooperationen ( $\$ 10$ ASV-RL)}

Zur Teilnahme an der ambulanten spezialfachärztlichen Versorgung von Patienten mit schweren Verlaufsformen onkologischer Erkrankungen nach Anlage 1 der ASV-RL ist der Abschluss einer Kooperationsvereinbarung nach § 116b Absatz 4 Satz 10 SGB V verpflichtend. Zweck der ASV-Kooperation ist die Förderung der intersektoralen Kooperation in diesem Versorgungsbereich ( $\$ 10$ Abs. 1 S. 4 ASV-RL). Daher regelt $\S 10$ Abs. 1 S. 2 ASV-RL, dass es sich bei den verpflichtenden ASV-Ko- 
operationen um intersektorale Kooperationen zwischen geeigneten Leistungserbringern handeln muss (z.B. Vertragsärzte und zugelassene Krankenhäuser).

Vertragliche Vereinbarungen über die ASV-Kooperation sind dem erweiterten Landesausschuss im Anzeigeverfahren ebenfalls vorzulegen, es sei denn, eine solche Kooperation kommt nach $\S 116 \mathrm{~b}$ Absatz 4 Satz 11 SGB V nicht zustande (vgl. § 10 Abs. 2 ASV-RL).

\section{Die Stellung der Radiologie im interdisziplinären Team}

Im Rahmen des Beschlüsse zur Tuberkulose und der gastrointestinalen Tumoren hat der G-BA entschieden, dass die organisatorische Einbindung des Fachgebietes der Radiologie als sog. hinzuzuziehendes Fachgebiet erfolgt. Diese Form der organisatorischen Beteiligung der Radiologie in der ASV wird aus den nachfolgenden Gründen als medizinisch bedenklich angesehen.

\section{Radiologie als hinzuzuziehendes Fachgebiet}

In $\S 3$ Abs. 2 Satz 7 ASV-RL werden die sog. hinzuzuziehenden Ärzte wie folgt definiert:

„Die hinzuzuziehenden Fachärztinnen und Fachärzte sind solche, deren Kenntnisse und Erfahrungen in Abhängigkeit vom jeweiligen Krankheitsverlauf typischerweise bei einem Teil der Patientinnen und Patienten ergänzend benötigt werden.“

Der ASV-RL ist nicht zu entnehmen, dass das Fachgebiet der Radiologie automatisch zu den hinzuzuziehenden Fachgebieten gehört. Im Gegenteil müssen die Kenntnisse und Erfahrungen des Radiologen zur Behandlung z.B. der Tuberkulose, wie auch der gastrointestinalen Tumoren, in der Regel eingebunden werden. Daher ist die Radiologie beispielsweise auch Hauptkooperationspartner im Darmzentrum nach den Vorgaben der Deutschen Krebsgesellschaft.

Insofern stellt sich die Frage, warum ein Fachgebiet, welches für die Diagnostik und Behandlung einer bestimmten Erkrankung regelmäßig benötigt wird, nicht Teil des Kernteams ist. Die Radiologie ist bisher im Verbund beispielsweise mit Hausärzten und zentrumsorientierten Strukturen (Oncocert, Deutsche Krebsge- sellschaft, Deutsche Krankenhausgesellschaft) intensiv eingebunden, da das klinische Spektrum der unmittelbaren (und eben nicht sekundären) radiologischen Patientenversorgung sehr viel größer ist als das anderer methodendefinierter Fachgebiete, wie z.B. der Labormedizin oder der Pathologie.

In Punkt 2.2.3.1. der tragenden Gründen zum Beschluss des G-BA zur Anlage 2 a) Tuberkulose und atypische Mykobakteriose vom 19.12.2013 wird die Zuordnung der Radiologie als hinzuzuziehendes Fachgebiet wie folgt begründet:

„Labormedizinische und radiologische Leistungen stellen wichtige Säulen in der Diagnostik und der Behandlung von Tuberkulosepatienten dar. In vielen Fällen werden diese Leistungen von Labormedizinerinnen und Labormedizinern und Radiologinnen und Radiologen erbracht. Der Umfang des direkten Patientenkontaktes findet dabei jedoch selten in dem Maße statt, wie sie der G-BA bei der Beschreibung der Ebene des Kernteams vorsieht. Insofern wurden diese beiden Fachdisziplinen der dritten Ebene (hinzuziehende Ärztinnen und Ärzte) zugeordnet.“

Diese Begründung in den tragenden Gründen zu dem Beschluss zur Tuberkulose, wonach Fachärzte für Radiologie und Labormedizin gleichermaßen nur einen geringen Arzt-Patienten-Kontakt aufweisen und daher der Gruppe der hinzuzuziehenden Ärzte zugeordnet wurden, ist sachwidrig, da sie die tatsächliche Arbeitsweise des Radiologen verkennt. Für das Fachgebiet der Radiologie ist die Notwendigkeit des Arzt-Patienten-Kontakts im Bereich der Diagnostik eindeutig vorgeschrieben.Zudem ist der Radiologe aber auch therapeutisch tätig, sodass eine Gleichsetzung der Radiologie mit dem Fachgebiet der Labormedizin, welches nicht patientenorientiert arbeitet, nicht sachgerecht ist (zur Abgrenzung vgl. BSG, Urt. v. 05.11.1997, Az.: 6 RKa 52/97).

Der Ausschluss aus dem Kernteam und die Einstufung als ausschließlich hinzuzuziehendes Fachgebiet nach $\S 3$ Abs. 2 ASV-RL führt in Verbindung mit der Anordnung der Überweisung durch ein Mitglied des Kernteams im Rahmen eines „Definitionsoder Indikationsauftrages“ ( $§ 2$ Abs. 4 S. 2 ASV-RL) zudem dazu, dass der Radiologe in der ASV in die Diagnostik und Therapie der gastrointestinalen Tumoren und der Tuberkulose nicht mehr unmittelbar ein- gebunden ist und auch keine eigenen Therapieentscheidungen mehr treffen kann. Dies wird auch dadurch deutlich, dass keine Teilnahme des Radiologen an den Tumorkonferenzen in der ASV für die genannten Indikationen vorgesehen ist.

Nach der Definition in $\S 3$ Abs. 2 S. 1 ASVRL erfolgt die Hinzuziehung des Radiologen für die betreffenden Krankheiten durch das Kernteam „bei medizinischer Notwendigkeit" und darüber lediglich „zeitnah“. Dies bedeutet, dass es im Ermessen des Kernteams steht, ob eine medizinische Notwendigkeit zur Hinzuziehung des Radiologen bejaht wird. Eine Möglichkeit zur Mitentscheidung des Radiologen über die Durchführung diagnostischer Maßnahmen besteht hingegen nicht. Der Begriff der „zeitnahen Hinzuziehung“ macht deutlich, dass eine Beteiligung des Radiologen zu Beginn der Behandlung nicht erfolgen muss, sondern auch zu einem späteren Zeitpunkt möglich ist. Hierdurch wird die therapiebegleitende radiologische Diagnostik in einem unangemessenen Maße relativiert.

Diese Tendenz ist jedoch aus der Sicht der Radiologie sehr bedenklich, da sich jede Therapie auf eine gesicherte Diagnose stützen muss. Dabei spielt das Fachgebiet der Radiologie bereits zu Beginn der Therapie die zentrale, qualitätssichernde Rolle.

Seitens der Deutschen Röntgengesellschaft besteht die Befürchtung, dass das Fachgebiet der Radiologie, im Rahmen der Ausgestaltung der personellen Anforderungen ( $\S 3$ ASV-RL) und des Behandlungsumfanges ( $\S 5$ ASV-RL), im Bereich der gynäkologischen Tumoren und damit auch beim Mammakarzinom erneut lediglich als hinzuzuziehendes Fachgebiet aufgenommen wird. Eine nur mittelbare Beteiligung der Radiologie an den leitenden Therapieentscheidungen im Bereich der gynäkologischen Tumoren und insbesondere des Mammakarzinoms ist jedoch sehr fragwürdig, da das Fachgebiet der Radiologie, neben der Gynäkologie, in die Diagnostik und Therapie des Mammakarzinoms an erster Stelle einzubinden ist. Dies erscheint umso problematischer, als es sich hier um schwere Verlaufsformen des Mammakarzinoms handelt, die eine frühzeitige interdisziplinäre Diagnostik und Therapie erfordern.

Für den Bereich der gynäkologischen Tumoren ist auf die besondere Rolle der Ra- 
diologie im Bereich der Diagnostik und Therapie des Mammakarzinoms hinzuweisen. Dies gilt einerseits für das Mammografie-Screening, wo sich der Gesetzgeber dazu entschieden hat, in der Anlage 9.2 BMV-Ä den Radiologen neben dem Gynäkologen im Rahmen der Primärdiagnostik ohne Überweisungsvorbehalt einzubinden. Die Diagnostik des Mammakarzinoms gehört auch zum Kernbereich der Radiologie nach $\S 135$ Abs. 2 SGB V, da nach der Qualitätssicherungsvereinbarung zur kurativen Mammografie (Mammografie-Vereinbarung) gemäß § 3 ausschließlich Radiologen und Gynäkologen zur Diagnostik in der GKV berechtigt sind (vgl. hierzu für den Bereich der MRT: BVerfG Beschl. v. 8. 7. 2010, Az.: 2 BvR 520/07). Die Qualitätssicherungsvereinbarungen nach $\S 135$ Abs. 2 SGB V gelten für die ASV gemäß § 4 Abs. 2 ASV-RL entsprechend. Diese Regelungen belegen zudem, dass der Radiologe bei dieser Erkrankungsform nach der Legaldefinition in $\S 3$ Abs. 2 S. 3 ASV-RL zum Kernteam gehört, denn seine Kenntnisse und Erfahrungen zur Diagnostik und Behandlung des Mammakarzinoms müssen ,in der Regel eingebunden werden“.

Die Tatsache, dass der Radiologe Hauptbehandlungspartner im Bereich des Mammakarzinoms ist, ergibt sich auch aus der interdisziplinären S3-Leitlinie für die Diagnostik, Therapie und Nachsorge des Mammakarzinoms (AWMF-RegisterNummer: 032 - 045OL). Hier ist insbesondere folgender Umstand deutlich geworden: Da die ASV unverständlicherweise nicht die Nachsorge des Mammakarzinoms umfasst, muss diese im Rahmen der Regelversorgung ambulant oder stationär durchgeführt werden. Wenn der Radiologe jedoch vorher nicht in den Behandlungsprozess in der ASV einbezogen war (insbesondere keine Teilnahme an den Tumorkonferenzen) ist ihm eine sachgerechte Therapieentscheidung im Rahmen der Nachsorge voraussichtlich nicht möglich.

\section{Überweisung als Auftragsleistung} Medizinisch bedenklich erscheint auch die generelle Anordnung eines „Definitions- oder Indikationsauftrages“ für die hinzuzuziehenden Fachgebiete nach $\S 2$ Abs. 4 S. 2 ASV-RL. Diese Überweisungsart ist der Regelung in $\S 24$ BMV-Ä aus dem Bereich der vertragsärztlichen Versorgung entnommen. Im Gegensatz zu § 2 Abs. 4 S. 2 ASV-RL unterscheidet jedoch $\S$
24 Abs. 3 BMV-Ä zwischen folgenden Überweisungsarten, die arzt- und therapiebezogen eingesetzt werden können:

1. Zur Auftragsleistung oder

2. zur Konsiliaruntersuchung oder

3. zur Mitbehandlung oder

4. zur Weiterbehandlung

Bei der Auftragsleistung verbleibt die Verantwortung für Art und Umfang der angeforderten Leistung ausschließlich beim Auftraggeber. Der Auftragnehmer kann daher sein Leistungsspektrum und den Leistungsumfang nicht selbst bestimmen, da er grundsätzlich an den Auftrag des überweisenden Arztes gebunden ist. Er darf davon nur in Ausnahmefällen eigenverantwortlich oder nach Rücksprache mit dem Auftraggeber durch Ergänzung des Überweisungstraggebers abweichen. Demgegenüber lassen die übrigen Überweisungsarten dem Auftragnehmer größere Freiheiten in der Auswahl und der Entscheidung in Diagnostik und Therapie. Diese Überweisungsarten sind jedoch im Rahmen der ASV-RL nicht vorgesehen.

Die Entscheidung, die Radiologie als hinzuzuziehendes Fachgebiet auf Überweisung mit Definitions- und Indikationsauftrag, statt im Wege der ebenfalls denkbaren Konsiliaruntersuchung oder Mitbehandlung in den Behandlungsprozess einzubeziehen, widerspricht den medizinischen Erfordernissen in der ASV, da gerade bei den schweren Verlaufsformen die Interdisziplinarität nicht früh genug beginnen kann.

\section{Ergebnis}

Die erfolgte Zuordnung des Fachgebietes der Radiologie zu den hinzuzuziehenden Fachgebieten in den Bereichen der Tuberkulose und der gastrointestinalen Tumoren sowie deren ausschließliche Möglichkeit der Inanspruchnahme im Rahmen eines Definitions- oder Indikationsauftrages sind medizinisch bedenklich. Eine entsprechende Einbindung der Radiologie bei gynäkologischen Tumoren und insbesondere im Bereich des Mammakarzinoms wäre daher nicht vertretbar, zumal in diesem Bereich der Radiologe zu den Kernfächern zählt.

Die Deutsche Röntgengesellschaft wird daher folgende Änderungen der bisherigen Beschlüsse zur Tuberkulose und zu den gastrointestinalen Tumoren sowie im Rahmen der noch vorzunehmenden Be- schlussfassung zu den gynäkologischen Tumoren gegenüber dem G-BA anregen:

1. Das Fachgebiet der Radiologie sollte im Rahmen der Diagnostik und Therapie dieser Erkrankungen in das Kernteam einbezogen werden.

2. Zumindest sollte bei bestimmten Behandlungsformen, je nach Schwere der Erkrankung, ein Wechsel in das Kernteam vollzogen und der Radiologe unmittelbar eingebunden werden.

3. Der Überweisungsvorbehalt ist zwingend in eine Konsiliaruntersuchung oder Mitbehandlung zu ändern, sodass der Radiologe im Rahmen der Diagnostik und Therapie schwerer Verlaufsformen der betreffenden Erkrankungen eigenständige Therapieentscheidungen treffen kann.

Im Ergebnis ist davon auszugehen, dass eine Übernahme des bisherigen Behandlungskonzepts der ASV-RL im Bereich der gynäkologischen Tumoren, insbesondere aber für das Mammakarzinom, nicht zielführend sein und unter erheblichen Qualitätsmängeln leiden wird. Auch da die radiologische Diagnostik und Therapie des Mammakarzinoms primär außerhalb der ASV innerhalb der Regelversorgung stattfinden soll, bedarf es eines einheitlichen Behandlungskonzepts für beide Sektoren. Gerade weil es sich in der ASV um Patientinnen mit schweren Verlaufsformen des Mammakarzinoms handelt, muss sichergestellt sein, dass die Kernfächer rechtzeitig und engmaschig eingebunden werden. Andernfalls entstehen Qualitätsmängel, die gerade durch die ASV verhindert werden sollen.

\section{Prof. Dr. Peter Wigge}

Rechtsanwalt,

Fachanwalt für Medizinrecht

Rechtsanwälte Wigge

Scharnhorststr. 40

48151 Münster

Telefon: (0251) 53595-0

Telefax: (0251) 53595-99

E-Mail: kanzlei@ra-wigge.de

www.ra-wigge.de 